\title{
The Role of EGFR Inhibitors in the Treatment of Metastatic Anal Canal Carcinoma: A Case Series
}

\author{
Muhammad W. Saif, ${ }^{1}$ Ewa Kontny, ${ }^{2}$ Kostas N. Syrigos, ${ }^{3,4}$ and Armin Shahrokni² \\ ${ }^{1}$ Division of Hematology and Oncology, Department of Medicine, Columbia University, New York, NY 10032-3784, USA \\ ${ }^{2}$ Griffin Hospital, Yale University School of Medicine, 130 Division Street, Derby, New Haven, CT 06418, USA \\ ${ }^{3}$ Division of Medical Oncology, Yale School of Medicine, 333 Cedar Street, New Haven, CT 06520, USA \\ ${ }^{4}$ Third Department of Medicine, Athens University School of Medicine, Building Z, Sotiria General Hospital, \\ Mesogion 15211527 Athens, Greece
}

Correspondence should be addressed to Kostas N. Syrigos, knsyrigos@usa.net

Received 17 January 2011; Revised 14 April 2011; Accepted 15 April 2011

Academic Editor: Bruno Vincenzi

Copyright (c) 2011 Muhammad W. Saif et al. This is an open access article distributed under the Creative Commons Attribution License, which permits unrestricted use, distribution, and reproduction in any medium, provided the original work is properly cited.

Anal cancer patients who have exhibited disease progression after having received all approved drugs pose a major therapeutic challenge. In addition to cytotoxic agents, novel targeted agents are being developed and have an established role in the treatment of many solid tumors, including colon cancer. However, their role in anal cancer is yet to be determined. Most anal malignancies are squamous cell carcinomas often strongly expressing epidermal growth factor receptors (EGFRs). Targeting the latter seems to result in favorable changes in tumor growth. We present three cases of refractory anal cancers, treated with EGFR inhibitors, after having received the recommended chemotherapy regimens. We conclude that EGFR inhibitors may play a vital role in the treatment of anal cancer and we suggest that large trials are be conducted in order to clarify their efficacy and to improve therapeutic management.

\section{Introduction}

Anal canal carcinoma (ACC) is a relatively rare gastrointestinal malignancy $[1,2]$, and its most common histological type is squamous cell carcinoma [3]. Five-year survival rates range from $78 \%$ in patients with local stage disease to only $18 \%$ in patients with distant metastases [1].

Historically, abdominoperineal resection (APR) was thought to be the standard treatment for nearly all anal cancers below the dentate line, with an approximate 70\% 5year survival [4]. The current standard treatment for invasive anal carcinoma is the combination of radiotherapy and chemotherapy. The 5-fluorouracil (5-FU) plus mitomycin C doublet combined with radiotherapy has been shown to be more effective than radiotherapy alone [5] as well as compared to radiotherapy plus 5-FU [6]. However, mitomycin C-related adverse events, such as hematological toxicity [7], often lead to discontinuation of this drug making it necessary to find less toxic but equally effective regimens. Other thera- peutic options, such as targeted agents, need to be explored.

The epidermal growth factor receptor (EGFR) (also known as HER-1 or c-erbB-1) is a glycoprotein that consists of an extracellular receptor, a transmembrane region, and an intracellular domain functioning as tyrosine kinase. There are 40,000 to 100,000 EGFR receptors per normal cell, whereas EGFR has been found overexpressed in most solid tumors, such as nonsmall cell lung, renal, ovarian, head and neck, and breast cancers [8]. It has been hypothesized that EGFR overexpression increases signal generation and activates downstream pathways making cells grow more aggressively and develop invasive characteristics [9]. There are two major categories of anti-EGFR therapeutics: antibodies binding to the extracellular ligand-binding region and small-molecule tyrosine-kinase inhibitors (TKIs) that compete with ATP for binding to the kinase domain [10]. The Food and Drug Administration (FDA) has approved the monoclonal antibodies cetuximab and panitumumab in the treatment of colorectal and head and neck cancer and 
erlotinib for lung and pancreatic cancer [10]. Finding EGFR overexpressed in ACC has triggered interest to investigate whether patients benefit from such targeted therapies.

In a study that examined tissue samples of 21 patients with ACC, it was found that all samples had 4+ EGFR expression while they were negative for HER-2 [11]. In another study concerning 38 squamous cell carcinomas of the anal canal ( 31 biopsies and 7 resection specimens) collected from 1989 to $2003,55 \%$ of tumors showed EGFR immunoreactivity. $62 \%$ of the latter had moderate to strong EGFR expression [12]. Since none of cases showed EGFR gene amplification, other mechanisms such as activating mutations, increased coexpression of receptor ligands, decreased receptor turnover, and heterodimerization with other heterologous receptor systems such as HER-2 might be involved [13].

We present three cases of ACC patients who were administered anti-EGFR therapy after having received the recommended chemotherapeutic regimens.

\section{Case Presentation (Table 1)}

2.1. Case 1. The first patient was a 73-year-old Caucasian female referred to us for salvage therapy. The patient had recurrent squamous cell carcinoma of the anorectal junction with lymph node metastases. The disease had recurred after cisplatin, 5-FU, and radiation therapy. The computed tomography scan (CT) showed abdominal as well as inguinal lymphadenopathy. Carcinogenic embryonic antigen (CEA) levels were $317 \mathrm{ng} / \mathrm{mg}$. After giving a fully informed consent, she was administered cetuximab at an initial dose of $400 \mathrm{mg} / \mathrm{m}^{2}$ followed by weekly doses of $250 \mathrm{mg} / \mathrm{m}^{2}$. In addition, she received mitomycin $\left(10 \mathrm{mg} / \mathrm{m}^{2}\right)$ on day 1 of each 28-day cycle. With the exception of a grade 2 cetuximabrelated rash, the doublet was well-tolerated until week 12 when mitomycin had to be stopped due to severe thrombocytopenia and hemolytic-uremic syndrome (HUS). According to new CT scans, the disease was stable (with a $17 \%$ decrease in the size of the abdominal lymph nodes) and CEA levels dropped to $214 \mathrm{ng} / \mathrm{mg}$. After the HUS was resolved, therapy was continued for an additional 4 cycles ( 8 weeks) but a magnetic resonance imaging (MRI) scan (CT was not performed due to concerns about contrast affecting renal function following recent HUS) revealed a 30\% increase in the left inguinal mass. CEA levels reached $1370 \mathrm{ng} / \mathrm{ml}$. We added irinotecan to the regimen and this doublet kept the disease stable for 11 cycles (5.5 months) up until an inguinal lymph node started to become enlarged and painful. The patient underwent palliative radiation therapy and later received modified FOLFOX-7 regimen. She died 3 months later.

2.2. Case 2. A 64-year-old Caucasian male presented to our clinic with the diagnosis of anal cancer with hepatic, lymph node and bone metastases. His disease had progressed after 1st line treatment of 5-FU, mitomycin $\mathrm{C}$ and radiation therapy and 2nd line cisplatin plus irinotecan. Cetuximab was offered as salvage therapy at an initial dose of $400 \mathrm{mg} / \mathrm{m}^{2}$ followed by weekly doses of $250 \mathrm{mg} / \mathrm{m}^{2}$ and the patient received 8 weekly courses. The treatment was generally welltolerated with the patient experiencing nausea grade 2, diarrhea grade 1 and rash grade 2. Restaging CT scans and bone scanning revealed that the disease was stable but the bone metastases' pain had increased. The patient chose to continue with palliative radiation therapy.

2.3. Case 3. The third patient was a 51-year-old African American male who had received ACC initial treatment consisting of mitomycin $\mathrm{C}, 5-\mathrm{FU}$ and radiation therapy. The disease recurred 1.5 years later, and the patient received FOLFOX with further progression to abdominal lymph nodes. A severe hypersensitivity reaction was observed during the initial infusion of cetuximab, and the patient was offered panitumumab monotherapy $(6 \mathrm{mg} / \mathrm{kg})$. After receiving four doses, he developed a grade 3 rash. He was treated with emollients, oral minocycline, and the dose was reduced to $4.5 \mathrm{mg}$. This dose was well tolerated, and we reescalated back to the full dose of $6 \mathrm{mg}$ within the next week. Disease progression was noted after a total of 12 cycles of panitumumab treatment (6 months).

\section{EGFR and KRAS Status}

EGFR status was retrospectively tested by immunostaining, and KRAS genotyping was performed by using polymerase chain reaction (PCR) with exon 1 flanking primers followed by single-strand conformational polymorphism (SSCP) analysis. All three patients had EGFR-positive tumors with different levels of EGFR expression (markedly stronger expression in the third patient), and no bandshifts in KRAS codons 12 and 13 were identified. All patients were found to have "wild-" type KRAS.

\section{Discussion}

In all three cases, we were able to show variable diseasefree survival (from 8 to 24 weeks) after all recommended regimens for advanced ACC had failed. This wide range may be attributed to different KRAS/EGFR expression levels, which were not routinely checked at that time. The first patient received EGFR-targeted therapy concurrently with chemotherapy. However, in the third patient, the disease was kept stable with EGFR-targeted monotherapy.

Existing data concur that EGFR is a valid therapeutic target in ACC as most of these carcinomas are of squamous cell histology and often strongly express EGFR. Its inhibition seems to result in favorable tumor growth changes [11]. Most interestingly, Paliga et al. showed that squamous cell anal canal carcinoma lacks the most common K-ras and EGFR mutations, suggesting that cetuximab may enhance radiosensitivity. The investigators evaluated the mutation status of KRAS exon 2 and EGFR exon 19 and 21 in 95 tumor biopsies before treatment. They found that KRAS exon 2 mutations and EGFR exon 19 mutations were absent in all 95 samples. Only 3 of the samples scored positive for EGFR exon 21 [14]. Another important study was the one conducted by Van Damme et al. [15]. They reported absence of Kras (exon 2) and EGFR mutations (exon 18, 20) in ACC. EGFR expression was present in $83.7 \%$ of interpretable cases whereas there was no EGFR amplification. They concluded 
TABLE 1: Details on the treatment of the three ACC patients receiving anti-EGFR agents.

\begin{tabular}{|c|c|c|c|c|c|c|c|c|c|c|c|}
\hline $\mathrm{Pt}$ & $\begin{array}{l}\text { Location at } \\
\text { diagnosis }\end{array}$ & 1st line & Rec site & $\begin{array}{l}\text { TTP } \\
\text { (wks) }\end{array}$ & 2nd line & Rec site & $\begin{array}{l}\text { TTP } \\
\text { (wks) }\end{array}$ & 3rd line & Rec site & $\begin{array}{l}\text { TTP } \\
\text { (wks) }\end{array}$ & $\begin{array}{l}\text { Treatment } \\
\text { after } \\
\text { anti-EGFR } \\
\text { agent }\end{array}$ \\
\hline 1 & $\begin{array}{l}\text { Anorectal } \\
\text { junction- } \\
\text { iliac lymph } \\
\text { nodes }\end{array}$ & $\begin{array}{l}\text { Cis-5-FU- } \\
\text { RT }\end{array}$ & $\begin{array}{l}\text { Abdominal, } \\
\text { inguinal LN }\end{array}$ & 5 & $\begin{array}{l}\text { Cetux- } \\
\text { MMC-10 } \\
\text { courses (week } \\
12: \text { MMC } \\
\text { discontinued) }\end{array}$ & $\begin{array}{l}\text { Left } \\
\text { inguinal } \\
\text { mass }\end{array}$ & 20 & $\begin{array}{l}\text { Cetux-Iri } \\
\text { (11 courses) }\end{array}$ & $\begin{array}{l}\text { inguinal } \\
\text { LN }\end{array}$ & 22 & $\begin{array}{l}\text { RT-modified } \\
\text { FOLFOX-7- } \\
\text { the patient } \\
\text { died } 3 \\
\text { months later }\end{array}$ \\
\hline 2 & $\begin{array}{l}\text { Rectum, } \\
\text { liver, } \\
\text { abdominal } \\
\text { LN, bones }\end{array}$ & $\begin{array}{l}\text { 5-FU- } \\
\text { MMC }\end{array}$ & Liver & 6 & Cis plus Iri & $\begin{array}{l}\text { Liver, bone } \\
\text { metastases }\end{array}$ & 8 & $\begin{array}{l}\text { Cetux } \\
(8 \text { courses })\end{array}$ & $\begin{array}{l}\text { Skeletal } \\
\text { pain } \\
\text { increased }\end{array}$ & 8 & $\begin{array}{l}\text { Palliative RT } \\
\text { for bone } \\
\text { pain-no PD } \\
\text { visible in } \\
\text { scanning or } \\
\text { CT-patient } \\
\text { did not wish } \\
\text { to continue } \\
\text { treatment }\end{array}$ \\
\hline 3 & $\begin{array}{l}\text { Anal } \\
\text { sphincter } \\
\text { T3N0M0 }\end{array}$ & $\begin{array}{l}\text { MMC, } \\
\text { 5-FU, and } \\
\text { RT }\end{array}$ & $\begin{array}{l}\text { Abdominal } \\
\text { lymph } \\
\text { nodes }\end{array}$ & 81 & FOLFOX & $\begin{array}{l}\text { Abdominal } \\
\text { LN }\end{array}$ & 5 & $\begin{array}{l}\text { Panitumumab } \\
\text { (after HSR to } \\
\text { cetuximab) } \\
\text { (12 courses) }\end{array}$ & $\begin{array}{l}\text { Local } \\
\text { recurrence }\end{array}$ & 6 & RT \\
\hline
\end{tabular}

Cetux: cetuximab, Cis: cisplatin, CT: computed tomography scans, HSR: hypersensitivity reaction, Iri: irinotecan, LN: lymph nodes, MMC: mitomycin C, PD: disease progression, Rec site: site of recurrence, RT: radiotherapy, wks: weeks.

that EGFR and KAS mutation analysis is not useful as a screening method for testing for sensitivity to anti-EGFR treatment in ACC. It is important to point out that the high expression of EGFR does not mean that the gene is functioning correctly, as it may still be mutated and its downstream pathway may be malfunctioning [15]. Based on the above, EGFR treatment was administered to our 3 patients without any information regarding EGFR and KRAS status and these were retrospectively tested. However, we must note that EGFR has also been found overexpressed in breast, renal, and ovarian cancer, but no EGFR targeting treatment has been found active in these malignancies [16]. Furthermore, ACC studies on the use of other EGFR targeting molecules, such as erlotinib and gefitinib, are very limited, possibly because of lack of EGFR somatic mutations in ACC and great toxicity shown in phase I trials [17].

As is the case with refractory EGFR-expressing metastatic colorectal cancer, EGFR inhibitors should only be used in patients with nonmutated (wild-type) KRAS. This was also shown in the Lukan et al. study: two out of seven patients harbored KRAS mutations, and these were the ones with progressive disease receiving cetuximab. In the remaining 5 patients, there was partial remission, minor remission, or no change lasting $\geq 6$ months after previous rapid tumor progression [18].

Further questions arise as to whether a combination therapy with EGFR inhibitors would be most beneficial and which exact combination would be more appropriate. Phan and Hoff were the first to report the case of a female patient with refractory anal cancer who achieved excellent response with the combination of cetuximab and irinotecan after disease progression with irinotecan monotherapy [19]. Our first patient concurrently received mitomycin/irinotecan and experienced the longest disease-free progression. This combination was used on the basis of previous data regarding activity of this regimen. The com- bined use of cetuximab and mitomycin has been found more effective than cetuximab monotherapy in cytotoxicity assays conducted in vitro, suggesting synergy between the two agents [20]. Combined intraperitoneal chemotherapy of panitumumab, mitomycin-C, and irinotecan also showed efficacy in colorectal carcinomatosis in vivo [21]. There is an ongoing phase II trial studying the use of the combination of cetuximab, irinitecan and mitomycin of colorectal cancer [22]. In our first case, cetuximab was originally combined solely with mitomycin, and irinotecan was not immediately added, as we anticipated possible increased toxicity. The patient did actually experience hemolytic-uremic syndrome, but the prolonged disease-free survival was notable. This was also initially observed in irinotecan-refractory colorectal cancer: the addition of cetuximab increased response rates as compared to rates observed with monotherapy (22.9 percent versus 10.8 percent) [23]. A randomized phase III trial that concerned locoregionally advanced head and neck cancer revealed survival benefit with concurrent use of cetuximab and radiation [24].

The third case is the one that shows the benefit from anti-EGFR agents more clearly. Panitumumab is a fully human monoclonal antibody that constitutes an important agent in the treatment of colorectal cancer. It seems that it exerts its antitumor activity with a different mechanism than cetuximab and it is associated with far less hypersensitivity 
reactions than the latter [25]. Rash grade 2 was observed in the first and second case, and rash grade 3 was reported in case 3. These findings correlate with studies already published that support that the rash severity can be used as a predictive marker of response to cetuximab [26] and panitumumab [27] treatment.

In this study, we did not perform any screening technique to study the possible infection of the neoplasms with human papillomavirus (HPV). However, we should note that there are data supporting a correlation between oncogenic HPV and EGFR expression. In the Walker et al. study [28], $96 \%$ of invasive HPV-infected squamous ACC displayed strong membrane immunoreactivity to EGFR expression, further supporting the possible role of EGFR inhibitors in ACC treatment. They also showed that HIV-positive status contributes in augmenting EGFR expression levels that are involved in carcinogenesis. Rare cases of ACC also have a spindle cell component, especially when differentiation is poor, and exclusion of anal melanoma or primary gastrointestinal mesenchymal tumour may be required [29]. EGFR expression seems to differ significantly between spindle and epithelioid cells, as has been shown in vitro as well as in human studies [30], and this should also be taken into account when administering anti-EGFR treatment.

We should point out that in the three cases described above the patients received completely different EGFRtargeting regimens. The disease course in all three suggested that anti-EGFR agents are effective in ACC, but studies need to be performed to examine their usefulness as monotherapy in ACC salvage therapy as well as in specific combinations with cytotoxic agents and radiation therapy as first-line treatment. Large randomized studies are necessary in order to establish protocols. These trials must also include assessment of EGFR and KRAS mutations of the tissue specimens. Conformed.

\section{Disclosure}

Patient details have been removed to ensure anonymity. A consent form has been signed by close relatives of all three patients. Helsinki declaration was conformed.

\section{References}

[1] L. G. Johnson, M. M. Madeleine, L. M. Newcomer, S. M. Schwartz, and J. R. Daling, "Anal cancer incidence and survival: the surveilance, epidemiology, and end results experience, 1973-2000," Cancer, vol. 101, no. 2, pp. 281-288, 2004.

[2] A. Jemal, R. Siegel, E. Ward, Y. Hao, J. Xu, and M. J. Thun, "Cancer statistics, 2009," CA: Cancer Journal for Clinicians, vol. 59, no. 4, pp. 225-249, 2009.

[3] B. J. Cummings, "Metastatic anal cancer: the search for cure," Onkologie, vol. 29, no. 1-2, pp. 5-6, 2006.

[4] B. M. Boman, C. G. Moertel, and M. J. O'Connell, "Carcinoma of the anal canal: a clinical and pathologic study of 188 cases," Cancer, vol. 54, no. 1, pp. 114-125, 1984.

[5] H. Bartelink, F. Roelofsen, F. Eschwege et al., "Concomitant radiotherapy and chemotherapy is superior to radiotherapy alone in the treatment of locally advanced anal cancer: results of a phase III randomized trial of the European organization for research and treatment of cancer radiotherapy and gastrointestinal cooperative groups," Journal of Clinical Oncology, vol. 15, no. 5, pp. 2040-2049, 1997.

[6] M. Flam, M. John, T. F. Pajak et al., "Role of mitomycin in combination with fluorouracil and radiotherapy, and of salvage chemoradiation in the definitive nonsurgical treatment of epidermoid carcinoma of the anal canal: results of a phase III randomized intergroup study," Journal of Clinical Oncology, vol. 14, no. 9, pp. 2527-2539, 1996.

[7] J. A. Ajani, K. A. Winter, L. L. Gunderson et al., "Fluorouracil, mitomycin, and radiotherapy vs fluorouracil, cisplatin, and radiotherapy for carcinoma of the anal canal: a randomized controlled trial," Journal of the American Medical Association, vol. 299, no. 16, pp. 1914-1921, 2008.

[8] R. S. Herbst and C. J. Langer, "Epidermal growth factor receptors as a target for cancer treatment: the emerging role of IMC-C225 in the treatment of lung and head and neck cancers," Seminars in Oncology, vol. 29, no. 1, pp. 27-36, 2002.

[9] S. P. Ethier, "Signal transduction pathways: the molecular basis for targeted therapies," Seminars in Radiation Oncology, vol. 12, no. 3, pp. 3-10, 2002.

[10] H. X. Chen, J. N. Cleck, R. Coelho, and J. E. Dancey, "Epidermal growth factor receptor inhibitors: current status and future directions," Current Problems in Cancer, vol. 33, no. 4, pp. 245-294, 2009.

[11] L. H. Lê, R. Chetty, and M. J. Moore, "Epidermal growth factor receptor expression in anal canal carcinoma," American Journal of Clinical Pathology, vol. 124, no. 1, pp. 20-23, 2005.

[12] G. Alvarez, A. Perry, B. R. Tan, and H. L. Wang, "Expression of epidermal growth factor receptor in squamous cell carcinomas of the anal canal is independent of gene amplification," Modern Pathology, vol. 19, no. 7, pp. 942-949, 2006.

[13] C. L. Arteaga, "Epidermal growth factor receptor dependence in human tumors: more than just expression?" Oncologist, vol. 7, no. 4, pp. 31-39, 2002.

[14] A. A. Paliga, G. Chong, R. Onerheim, and A. Spatz, "KRAS and EGFR gene mutation status in anal canal carcinoma," in Poster Presentation at Gastrointestinal Cancers Symposium of American Society of Clinical Oncology (ASCO), June 2010, http://www asco http://www.asco.org/ASCOv2/Meetings/Abst racts?\&vmview=abst_detail_view\&confID $=72 \&$ abstractID $=20$ http://www asco http://www.asco.org/ASCOv2/Meetings/Abst 56.

[15] N. Van Damme, P. Deron, N. Van Roy et al., "Epidermal growth factor receptor and K-RAS status in two cohorts of squamous cell carcinomas," BMC Cancer, vol. 10, article 189, 2010.

[16] H. W. Lo, "Nuclear mode of the EGFR signaling network: biology, prognostic value, and therapeutic implications," Discovery Medicine, vol. 10, no. 50, pp. 44-51, 2010.

[17] B. G. Czito, C. G. Willett, J. C. Bendell et al., "Increased toxicity with gefitinib, capecitabine, and radiation therapy in pancreatic and rectal cancer: phase I trial results," Journal of Clinical Oncology, vol. 24, no. 4, pp. 656-662, 2006.

[18] N. Lukan, P. Ströbel, A. Willer et al., "Cetuximab-based treatment of metastatic anal cancer: correlation of response with KRAS mutational status," Oncology, vol. 77, no. 5, pp. 293-299, 2009.

[19] L. K. Phan and P. M. Hoff, "Evidence of clinical activity for cetuximab combined with irinotecan in a patient with refractory anal canal squamous-cell carcinoma: report of a case," Diseases of the Colon and Rectum, vol. 50, no. 3, pp. 395-398, 2007. 
[20] W. Shao, S. Zhao, Z. Liu et al., "Inhibition of human tumor xenograft growth in nude mice by a conjugate of monoclonal antibody LA22 to epidermal growth factor receptor with antitumor antibiotics mitomycin C," Biochemical and Biophysical Research Communications, vol. 349, no. 2, pp. 816-824, 2006.

[21] M. S. Cohen, M. F. Al-Kasspooles, S. K. Williamson, D. Henry, M. Broward, and K. F. Roby, "Combination intraperitoneal chemotherapy is superior to mitomycin $\mathrm{C}$ or oxaliplatin for colorectal carcinomatosis in vivo," Annals of Surgical Oncology, vol. 17, no. 1, pp. 296-303, 2010.

[22] "A Phase II Study of Mitomycin C, Irinotecan and Cetuximab in Patients with Previously Treated, Metastatic Colorectal Cancer," 2010, http://www.clinicaltrials.gov/ct2/show/ NCT00271011?term=mitomycin+cetuximab\&rank=1.

[23] D. Cunningham, Y. Humblet, S. Siena et al., "Cetuximab monotherapy and cetuximab plus irinotecan in irinotecanrefractory metastatic colorectal cancer," The New England Journal of Medicine, vol. 351, no. 4, pp. 337-345, 2004.

[24] J. A. Bonner, P. M. Harari, J. Giralt et al., "Radiotherapy plus cetuximab for squamous-cell carcinoma of the head and neck," The New England Journal of Medicine, vol. 354, no. 6, pp. 567-578, 2006.

[25] M. W. Saif, K. Kaley, E. Chu, and M. S. Copur, "Safety and efficacy of panitumumab therapy after progression with cetuximab: experience at two institutions," Clinical Colorectal Cancer, vol. 9, no. 5, pp. 315-318, 2010.

[26] J. H. Park, S.-W. Han, D.-Y. Oh et al., "Analysis of KRAS, BRAF, PTEN, IGF1R, EGFR intron 1 CA status in both primary tumors and paired metastases in determining benefit from cetuximab therapy in colon cancer," Cancer Chemotherapy and Pharmacology. In press.

[27] C. Gravalos, J. Cassinello, P. García-Alfonso, and A. Jimeno, "Integration of panitumumab into the treatment of colorectal cancer," Critical Reviews in Oncology/Hematology, vol. 74, no. 1, pp. 16-26, 2010.

[28] F. Walker, L. Abramowitz, D. Benabderrahmane et al., "Growth factor receptor expression in anal squamous lesions: modifications associated with oncogenic human papillomavirus and human immunodeficiency virus," Human Pathology, vol. 40, no. 11, pp. 1517-1527, 2009.

[29] C. Faus, D. Roda, M. Frasson et al., "The role of the pathologist in rectal cancer diagnosis and staging and surgical quality assessment," Clinical and Translational Oncology, vol. 12, no. 5, pp. 339-345, 2010.

[30] A. G. M. Scholes, S. Hagan, P. Hiscott, B. E. Damato, and I. Grierson, "Overexpression of epidermal growth factor receptor restricted to macrophages in uveal melanoma," Archives of Ophthalmology, vol. 119, no. 3, pp. 373-377, 2001. 


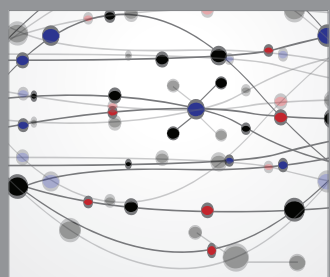

The Scientific World Journal
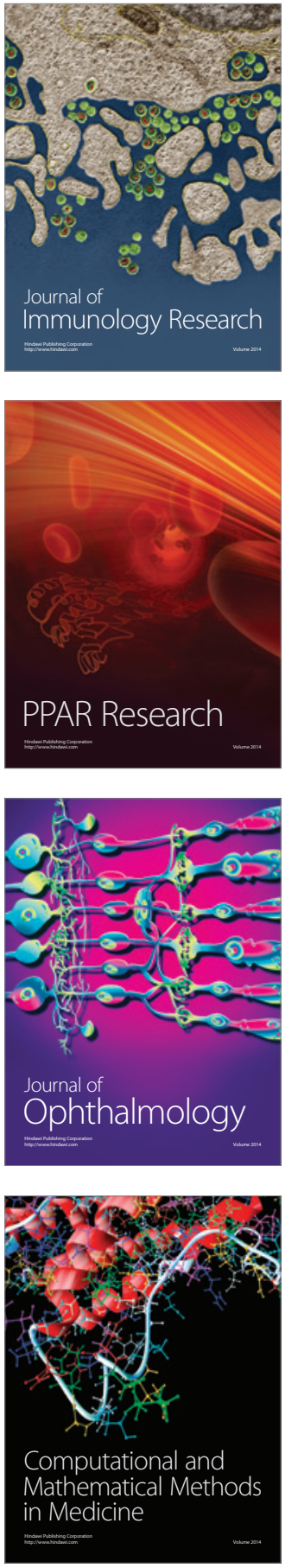

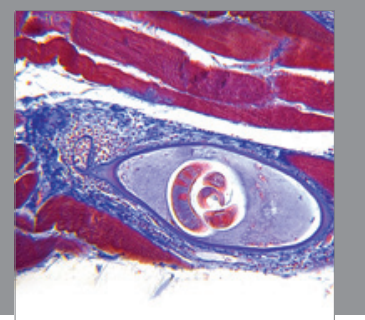

Gastroenterology

Research and Practice
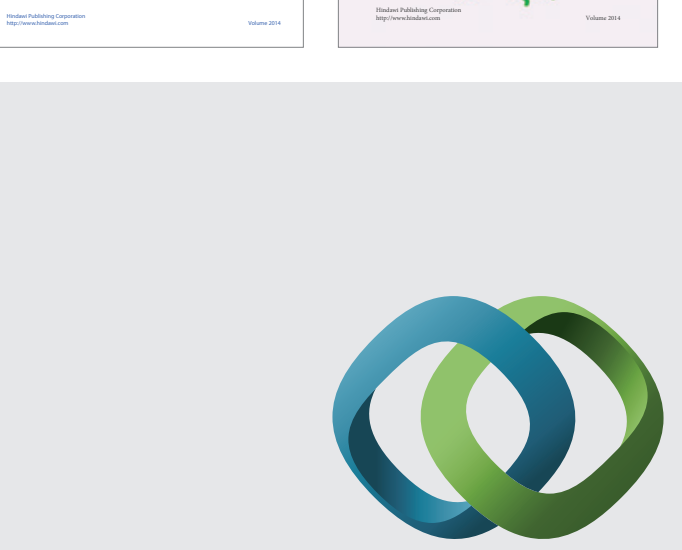

\section{Hindawi}

Submit your manuscripts at

http://www.hindawi.com
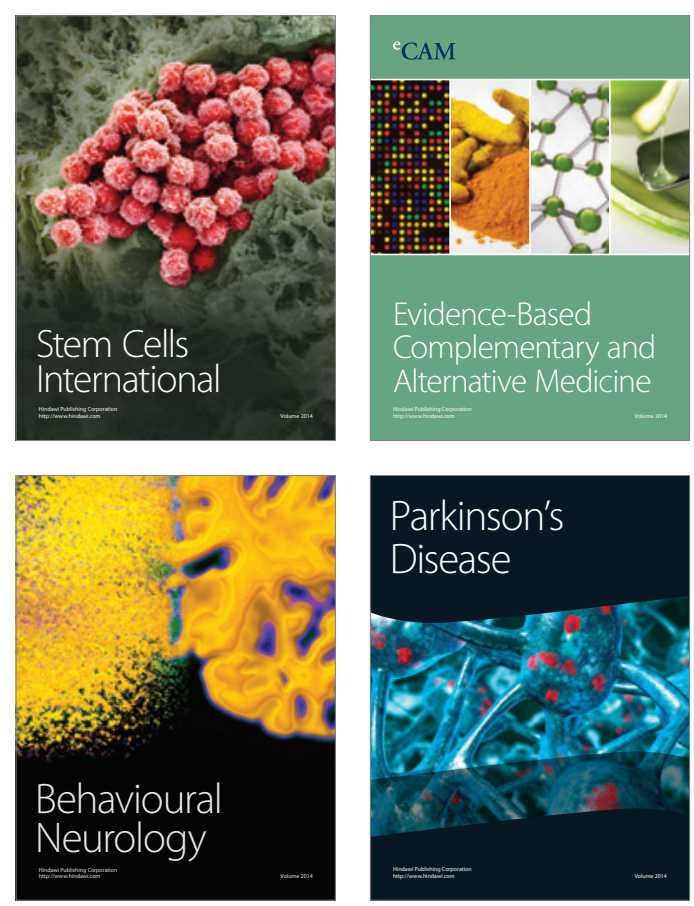

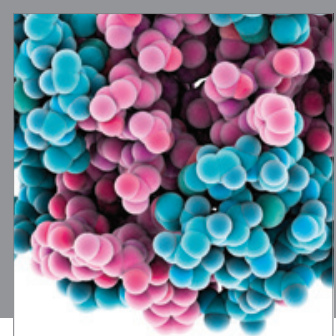

Journal of
Diabetes Research

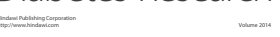

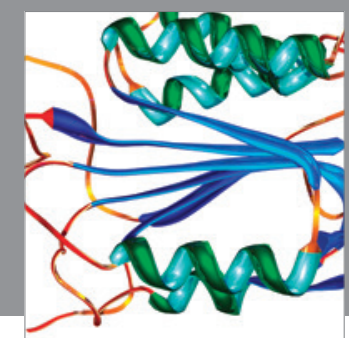

Disease Markers
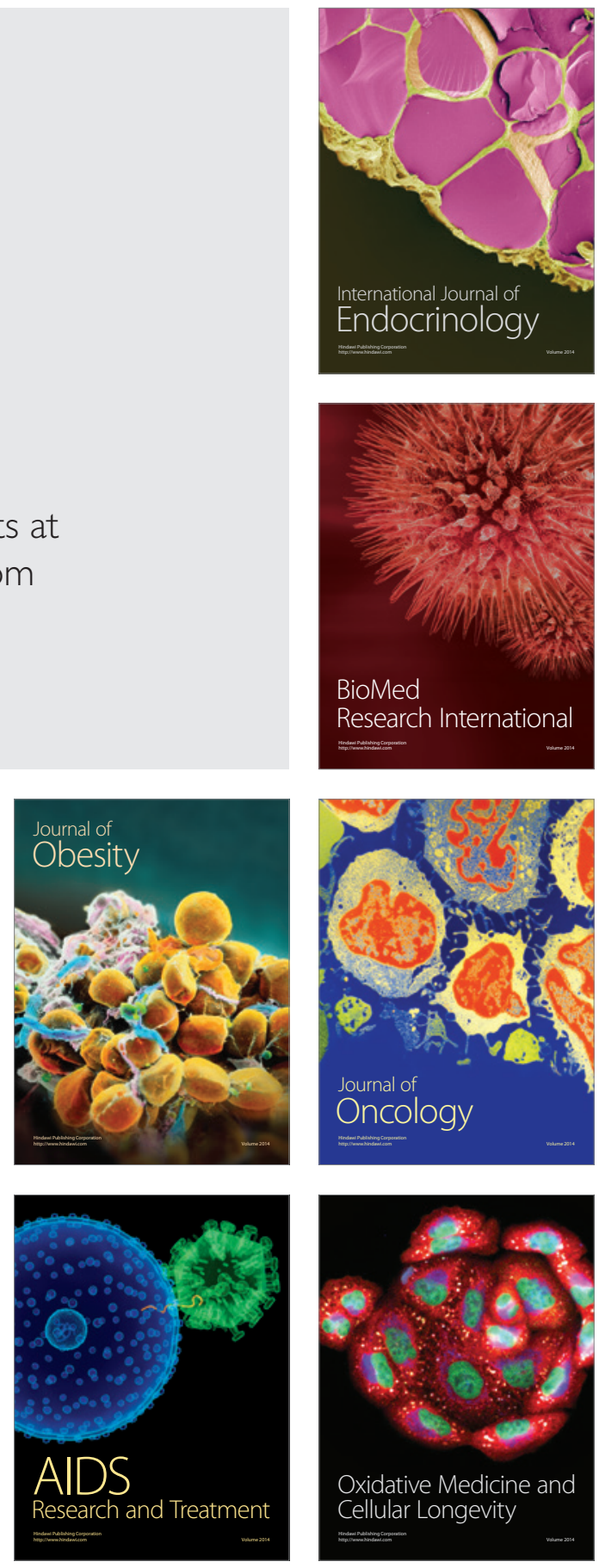